\title{
The global distribution of magnetic helicity in the solar corona ${ }^{\star}$ (Corrigendum)
}

\author{
A. R. Yeates ${ }^{1}$ and G. Hornig ${ }^{2}$ \\ ${ }^{1}$ Department of Mathematical Sciences, Durham University, Durham, DH1 3LE, UK \\ e-mail: anthony.yeates@durham.ac.uk \\ 2 Division of Mathematics, University of Dundee, Dundee, DD1 4HN, UK
}

A\&A 594, A98 (2016), DOI: 10.1051/0004-6361/201629122

Key words. magnetic fields - magnetohydrodynamics (MHD) - Sun: corona - Sun: magnetic fields - Sun: coronal mass ejections (CMEs) - errata, addenda

It has come to our attention that incorrect units were inadvertently used for the axes and colour scales of a number of figures in our paper (Yeates \& Hornig 2016). Specifically:

1. The units of field line helicity $\mathcal{A}$ are quoted as Maxwells [Mx], but the values for $\mathcal{A}$ shown in Figs. 3, 5, 7, $10,11 \mathrm{e}$ and 12 in the original paper were actually in $\mathrm{Mx} R_{\odot}^{-2}$. They should be multiplied by $\left(6.96 \times 10^{10}\right)^{2}$ to reach the true value in Mx.

2. The units of total helicity $H$, and of $\mathrm{d} H / \mathrm{d} t$ are quoted as $\mathrm{Mx}^{2}$, but the values were actually in $\mathrm{Mx}^{2} R_{\odot}^{-4}$. This applies to Figs. $4 \mathrm{c}, \mathrm{d}, 8 \mathrm{c}, \mathrm{d}$ and $11 \mathrm{~d}$. They should be multiplied by $\left(6.96 \times 10^{10}\right)^{4}$ to reach the true value in $\mathrm{Mx}^{2}$.

Corrected versions of the affected figures are included here. This does not in any way affect the qualitative results or conclusions of the paper, but we would like to correct the error for the benefit of future readers who may wish to make quantitative comparisons.

To put the corrected values into context, DeVore (2000) estimates that the typical helicity content of a single interplanetary magnetic cloud is of the order $2 \times 10^{42} \mathrm{Mx}^{2}$, which is similar to that shed in our axisymmetric flux rope (Fig. 8c). And Berger \& Ruzmaikin (2000) estimate a net transfer rate of helicity due to solar rotation of up to $5 \times 10^{43} \mathrm{Mx} \mathrm{day}^{-1}$ in each hemisphere, at solar maximum. This is also consistent with our results, although a more detailed comparison with a fully datadriven simulation remains to be performed in the future.

\section{References}

Berger, M. A., \& Ruzmaikin, A. 2000, J. Geophys. Res., 105, 10481 DeVore, C. R. 2000, ApJ, 539, 944

Yeates, A. R., \& Hornig, G. 2016, A\&A, 594, A98

\footnotetext{
* Movies associated to Figs. 10 and 12 are available at http://www . aanda.org
} 

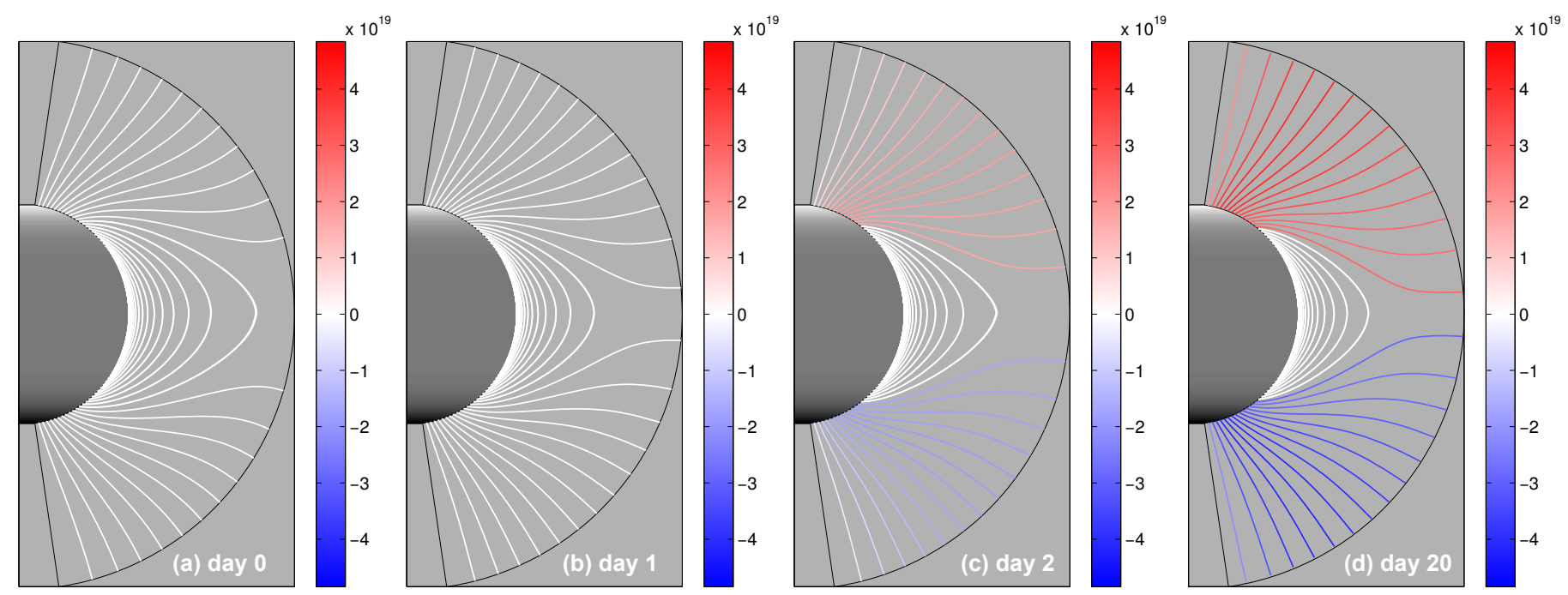

Fig. 3. Illustration of the dipolar simulation with $v_{0}=0.36 \times 10^{-5} \mathrm{~s}^{-1}$ on days $0,1,2$, and 20 . Greyscale shading on $r=r_{0}$ shows $B_{r}$ (white positive, black negative, saturated at $\pm 0.5 \mathrm{G}$ ), and projected coronal magnetic field lines traced from height $r=R_{\odot}$ are coloured (red/blue) according to $\mathcal{A}$, saturated at $\pm 5 \times 10^{19} \mathrm{Mx}$ with white indicating $\mathcal{A} \approx 0 \mathrm{Mx}$.
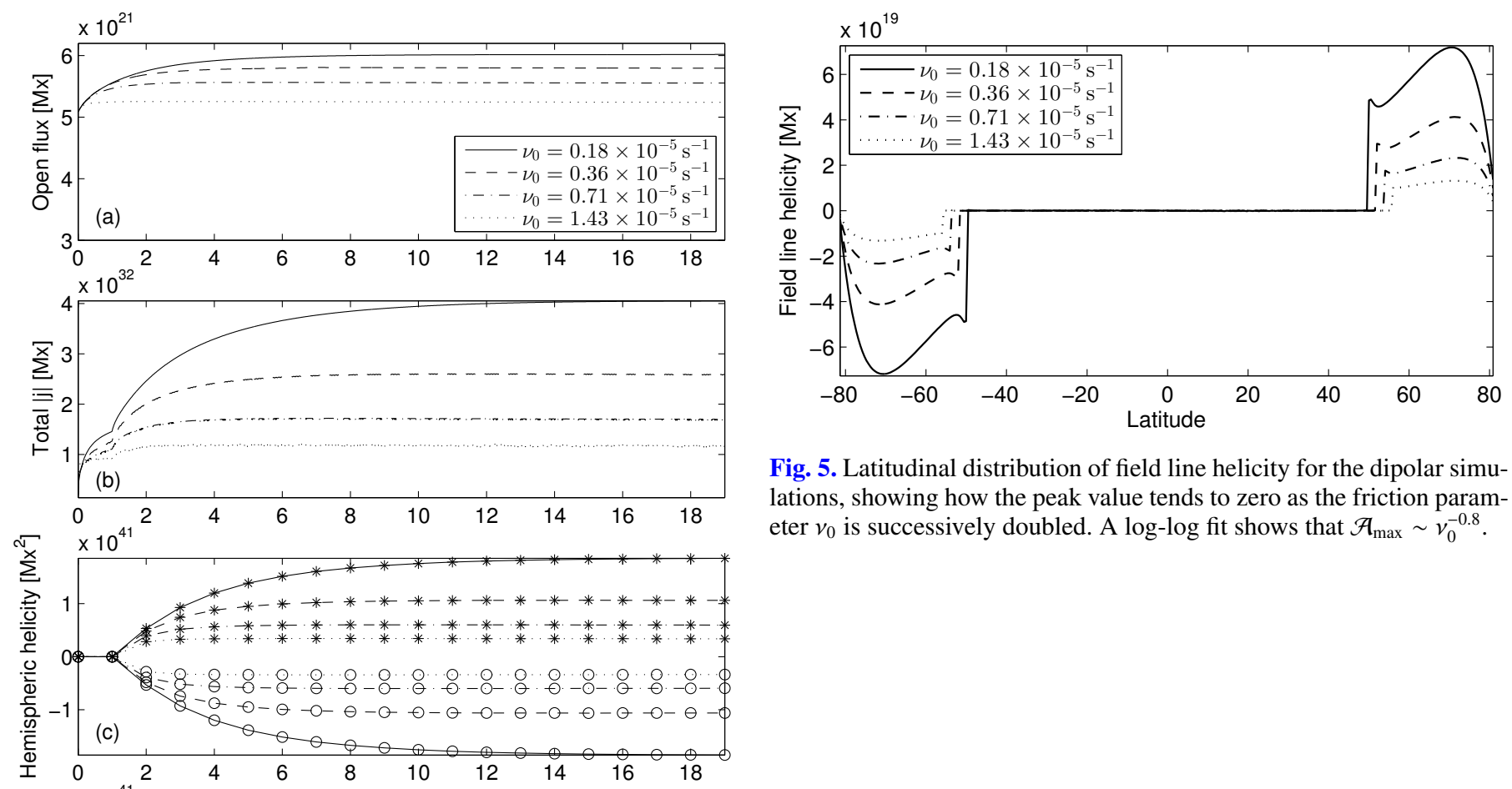

Fig. 5. Latitudinal distribution of field line helicity for the dipolar simulations, showing how the peak value tends to zero as the friction parameter $v_{0}$ is successively doubled. A $\log -\log$ fit shows that $\mathcal{A}_{\max } \sim v_{0}^{-0.8}$.

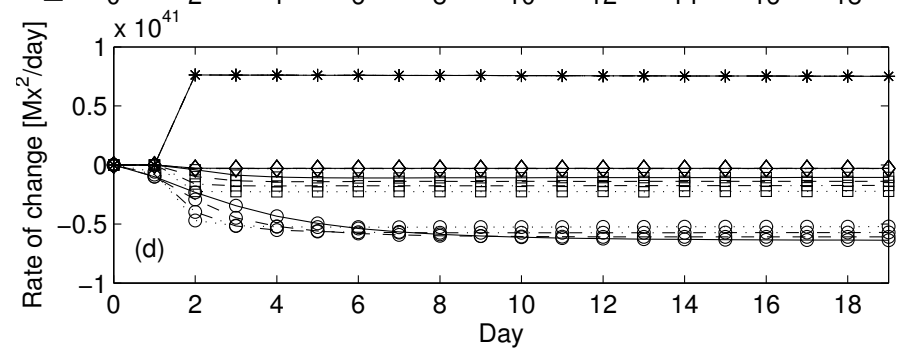

Fig. 4. Various integrated quantities as a function of time, for the dipolar simulations with different $v_{0}$ (indicated by line styles). Panel a) shows the open magnetic flux $\int_{r=r_{1}}\left|B_{r}\right| \mathrm{d} \Omega$, panel b) shows $\int_{D}|j| \mathrm{d} V$, and panel c) shows $H_{\mathrm{N}}$ (asterisks) and $H_{\mathrm{S}}$ (circles). Panel d) shows the terms in Eq. (18) for the northern hemisphere, with asterisks denoting $S_{0}$, circles $S_{1}$, squares $S_{\text {eq }}$, and diamonds $S_{V}$. 
A. R. Yeates and G. Hornig: The global distribution of magnetic helicity in the solar corona (Corrigendum)

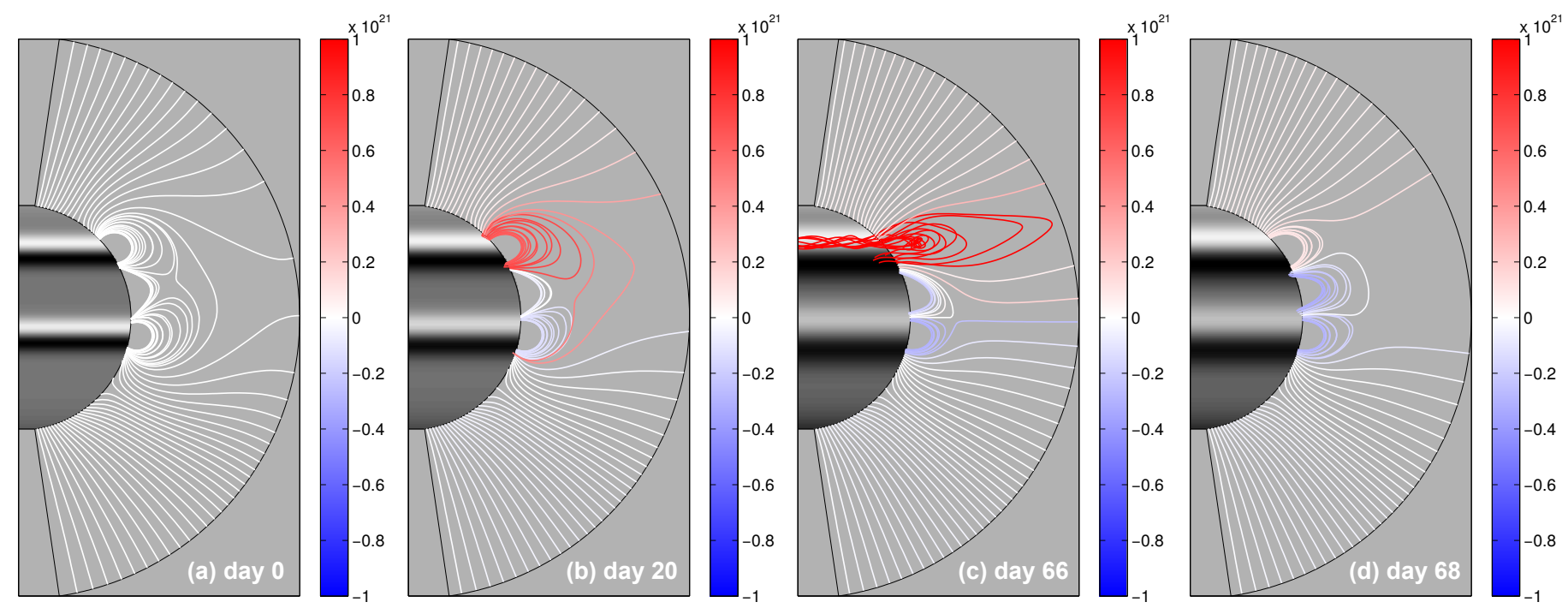

Fig. 7. Illustration of the quadrupolar simulation with $v_{0}=0.36 \times 10^{-5} \mathrm{~s}^{-1}$ on days $0,20,66$, and 68. Greyscale shading on $r=r_{0}$ shows $B_{r}$ (white positive, black negative, saturated at $\pm 2 \mathrm{G}$ ), and projected coronal magnetic field lines traced from height $r=1.2 R_{\odot}$ are coloured (red/blue) according to $\mathcal{A}$, saturated at $\pm 10^{21} \mathrm{Mx}$ with white indicating $\mathcal{A} \approx 0 \mathrm{Mx}$.
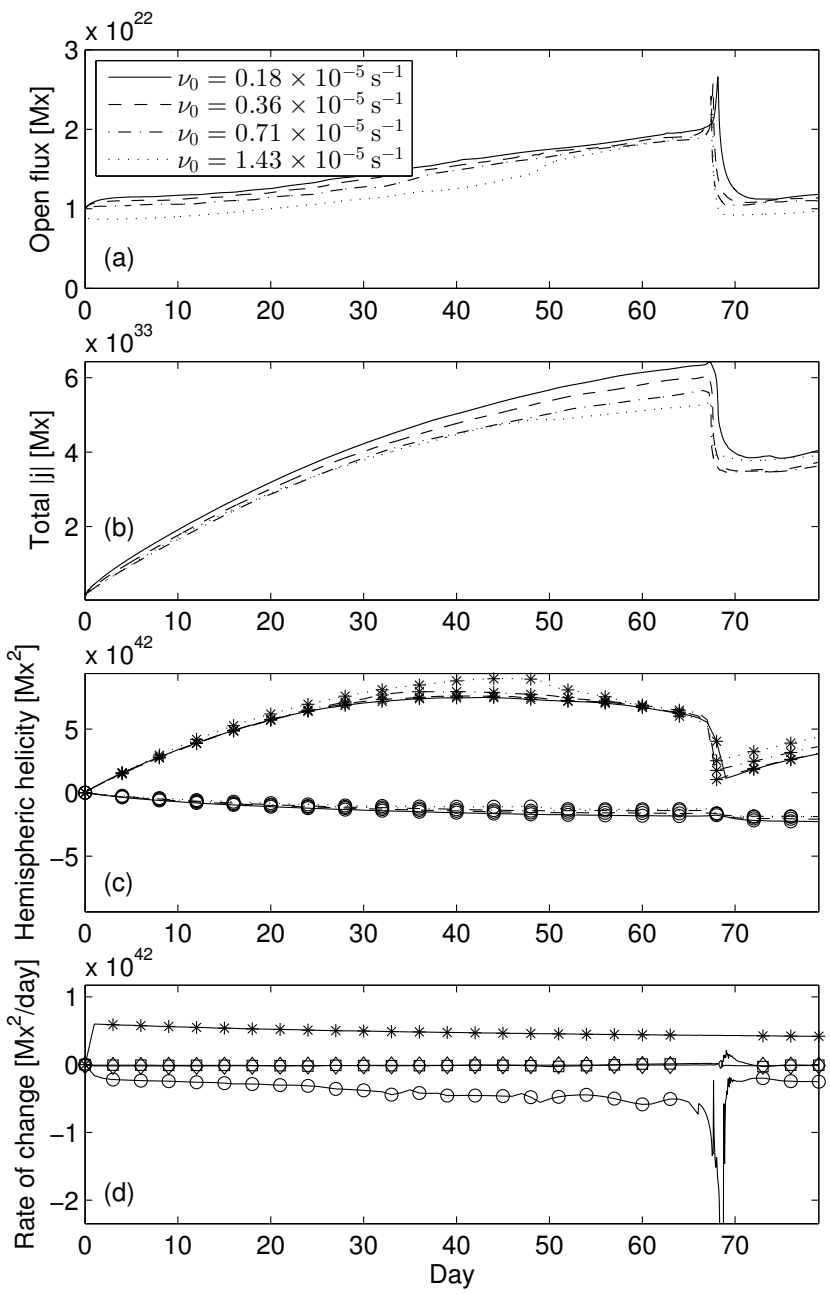

Fig. 8. Various integrated quantities as a function of time, for the quadrupolar simulations with different $v_{0}$ (indicated by line styles). The format is the same as Fig. 4. For clarity, panel d) shows only the run with $v_{0}=0.36 \times 10^{-5} \mathrm{~s}^{-1}$, and only for the northern hemisphere, although the hemispheres are no longer symmetric. The peak value of $S_{1}$ during the flux rope eruption is not shown, and is much larger, about $-2.7 \times 10^{43} \mathrm{Mx}^{2}$ day $^{-1}$. 

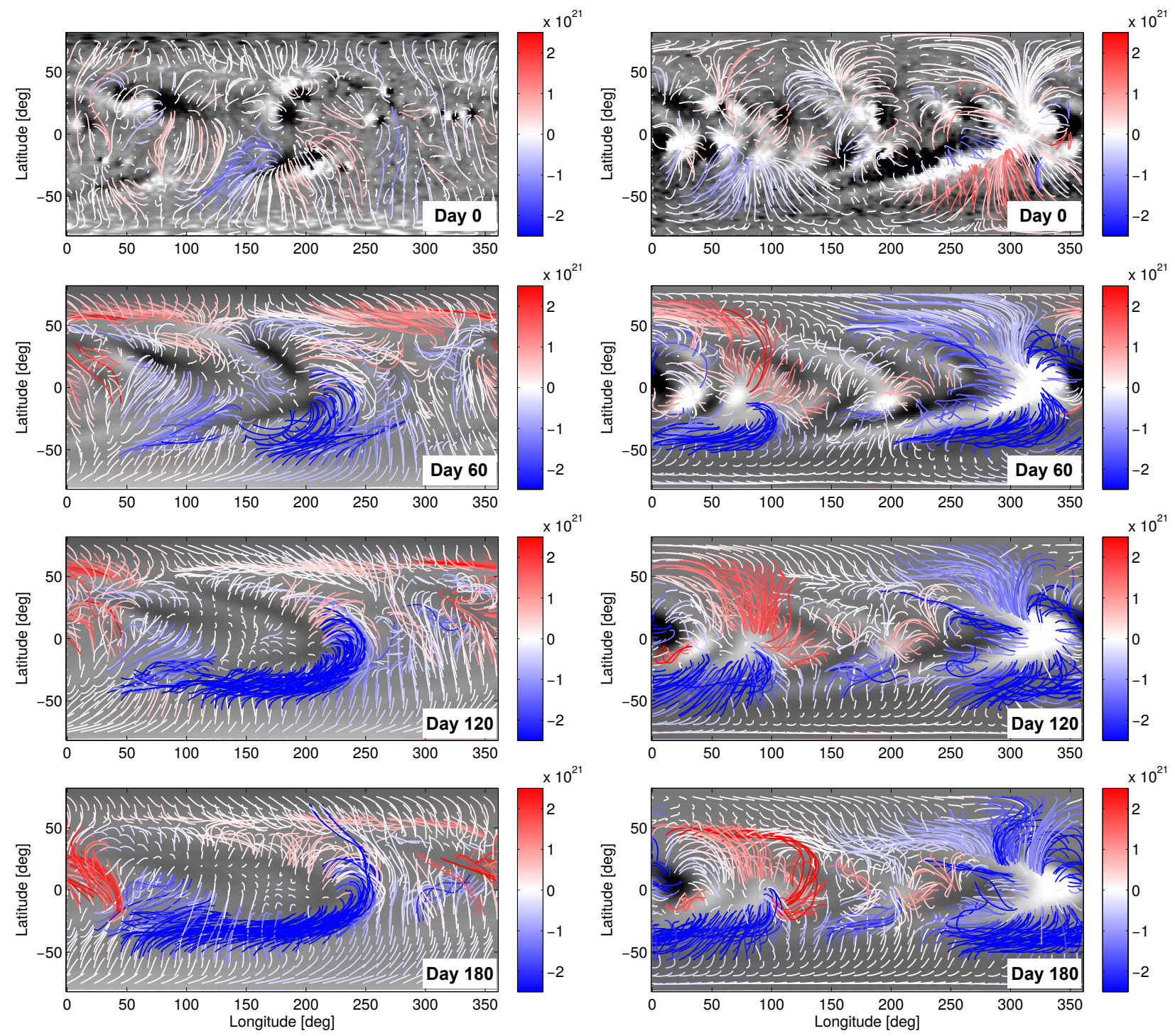

Fig. 10. Projected magnetic field lines in the period A (left column) and B (right column) simulations, on days 0, 60, 120, and 180. Greyscale shading on $r=r_{0}$ shows $B_{r}$ (white positive, black negative, saturated at $\pm 10 \mathrm{G}$ ), and projected coronal magnetic field lines traced from height $r=r_{0}$ are coloured (red/blue) according to $\mathcal{A}$, saturated at $\pm 2.5 \times 10^{21} \mathrm{Mx}$. Animated versions of these sequences are available online. 
A. R. Yeates and G. Hornig: The global distribution of magnetic helicity in the solar corona (Corrigendum)
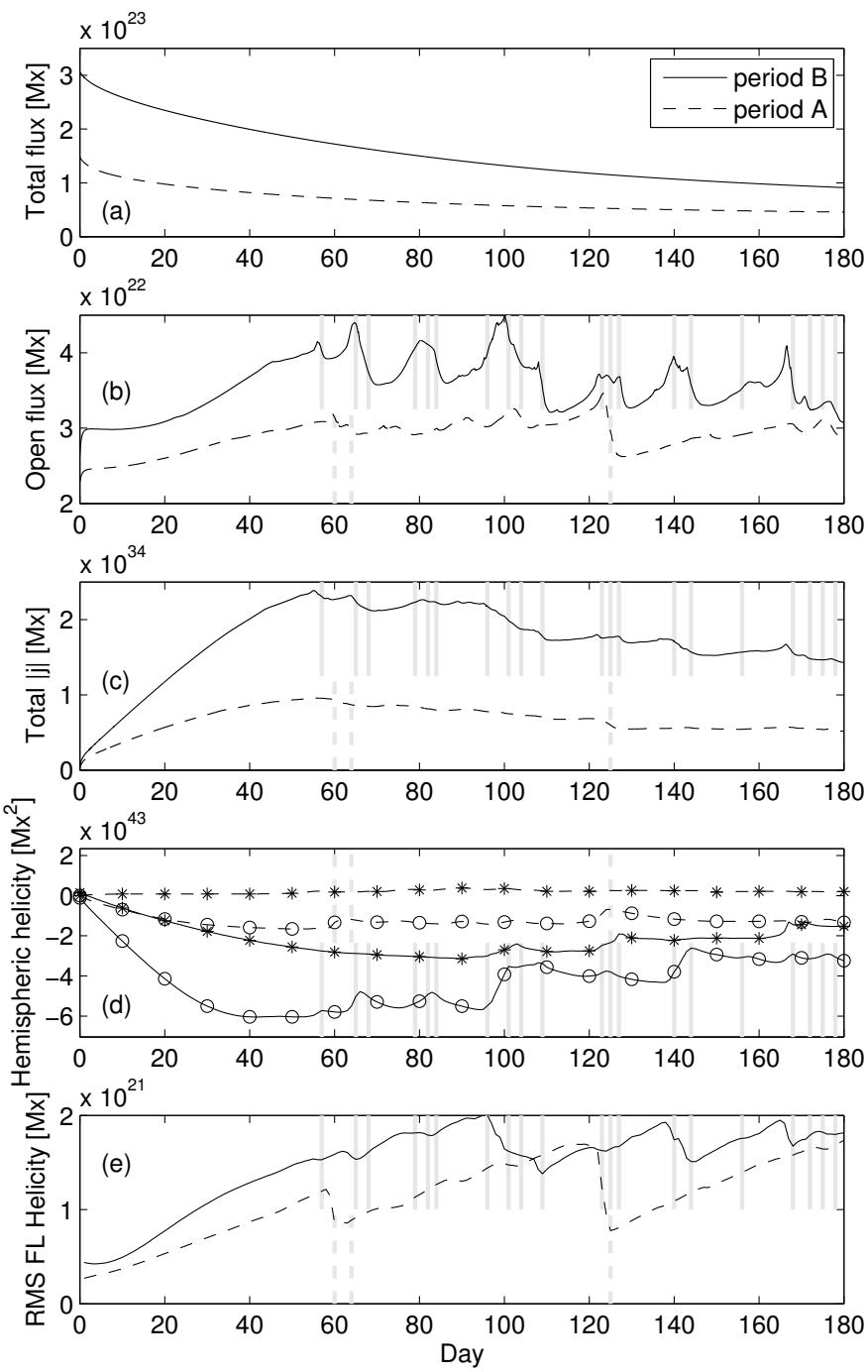

Fig. 11. Various integrated quantities as a function of time, for the nonaxisymmetric simulations (periods $\mathrm{A}$ and $\mathrm{B}$ ). Panel a) shows the total photospheric magnetic flux $\int_{r=r_{0}}\left|B_{r}\right| \mathrm{d} \Omega$, panel b) shows the total open flux $\int_{r=r_{1}}\left|B_{r}\right| \mathrm{d} \Omega$, panel c) shows $\int_{D}|j| \mathrm{d} V$, panel d) shows $H_{\mathrm{N}}$ (asterisks) and $H_{\mathrm{S}}$ (circles), and panel e) shows the root-mean-square field line helicity $\left(\int_{D} \mathcal{A}^{2} \mathrm{~d} V / \int_{D} \mathrm{~d} V\right)^{1 / 2}$. The vertical grey lines indicate times of strong flux rope ejections, as explained in the text. 

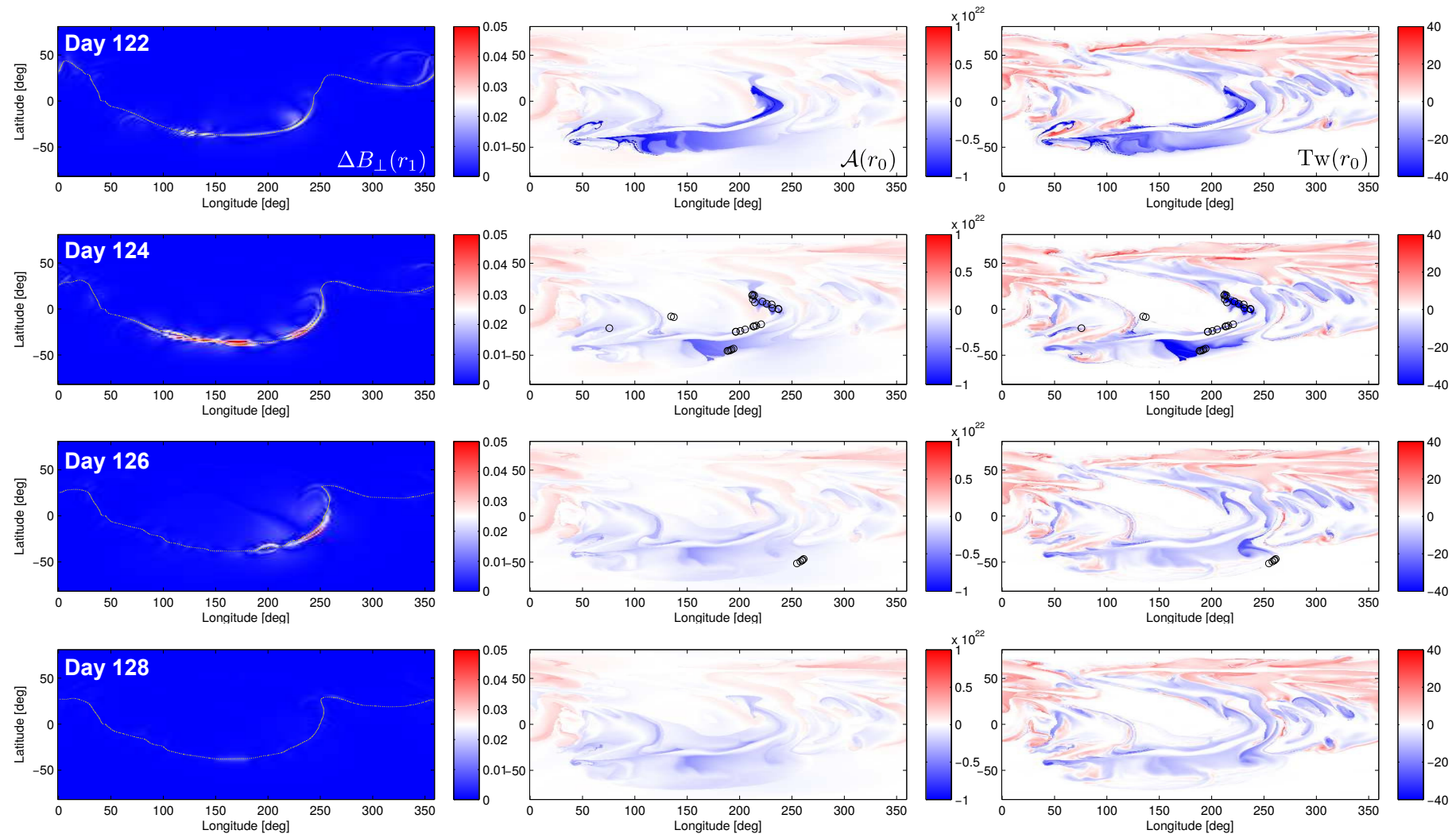

Fig. 12. Example of a flux rope ejection from period A. From top to bottom, the rows show days 122, 124, 126, and 128. The left column shows the (absolute) running daily difference of horizontal field $B_{\perp}:=\left(B_{\theta}^{2}+B_{\phi}^{2}\right)^{1 / 2}$ at the outer boundary $r=r_{1}$. The middle column shows the distribution of $\mathcal{A}$ on $r=r_{0}$ (saturated at $\pm 10^{22} \mathrm{Mx}$ ), and the right column shows the distribution of Tw at $r=r_{0}$ (saturated at \pm 40 ). The dashed lines in the left column show the neutral line where $B_{r}\left(r_{1}, \theta, \phi\right)=0$. Black circles in the other columns identify footpoints of field lines traced down from locations at $r=r_{1}$ where the running difference of $B_{\perp}$ exceeds $0.05 \mathrm{G} \mathrm{day}^{-1}$. Animated versions of this figure for both periods A and $\mathrm{B}$ are available online. 